Pathologe 2010 • [Suppl 2] 31:239-243

DOI 10.1007/s00292-010-1317-y

Online publiziert: 22. Juli 2010

(c) Springer-Verlag 2010

\author{
H. Moch \\ Institut für Klinische Pathologie, UniversitätsSpital Zürich, Schweiz
}

\section{Zystische Nierentumoren}

\section{Neue Tumortypen \\ und aktuelle molekulare Erkenntnisse}

Nierenzysten können im Zusammenhang mit Entwicklungsstörungen, erworbenen Nierenveränderungen und im Rahmen von Neoplasien entstehen. Nichtneoplastische zystische Läsionen schließen einfache Zysten, traumatische Zysten, infektiöse Zysten, Aneurysmen, aber auch Varixknoten ein. Entwicklungsanomalien mit zystischen Veränderungen finden sich bei kongenitalen Zystennieren des Neugeborenen oder des Erwachsenen. Sekundäre Zystenbildungen treten in wechselndem Ausmaß in Endstadium-Schrumpfnieren unter chronischer Hämodialyse und nach Nierentransplantation auf.

Zystische Nierentumoren sind per se kein eigener Tumortyp, aber viele Tumortypen weisen Zysten als kleinere Komponente, aber auch als dominantes makroskopisches Kriterium auf. Das papilläre Nierenzellkarzinom (vor allem Typ 1) kann radiologisch als große umkapselte Zyste, gefüllt mit hämorrhagischem bzw. nekrotischem Material imponieren. Auch Angiomyolipome können epitheliale Zysten aufweisen [1]. Unlängst wurden Onkozytome mit prominenten Zysten beschrieben [2]. Zystische Veränderungen sollen bei etwa $15 \%$ aller klarzelligen Nierenzellkarzinome auftreten. Manche klarzelligen Nierenzellkarzinome haben eine dominante zystische Komponente. In Einzelfällen können sie in einfachen Zysten entstehen. Das multilokuläre zystische Nierenzellkarzinom besteht ausschließlich aus Zysten.

In dieser Übersichtsarbeit werden folgende Nierentumoren mit Zysten als charakteristischen makroskopischen Befund beschrieben:
- zystisches Nephrom/gemischter Epithel- und Stromatumor,

- tubulozystisches Karzinom,

- synoviales Sarkom („cystic embryonal sarcoma"),

- Translokationskarzinome,

- Nierenzellkarzinome in EndstadiumNieren,

- klarzelliges Nierenzellkarzinom mit prominenten Zysten,

- multilokuläres zystisches Nierenzellkarzinom.

Weiterhin werden neue molekulare Befunde präsentiert, die ein molekulares Konzept der Nierentumorentstehung über einen zystenabhängigen und zystenunabhängigen Entstehungsmechanismus postulieren $[3,4]$.

\section{Gemischte Epithel- und Stromatumoren/ zystisches Nephrom}

Die gemischten Epithel- und Stromatumoren („mixed epithelial and stromal tumor“, MEST) der Niere wurden anfänglich unter dem Begriff „zystisches Hamartom des Nierenbeckens" beschrieben [5]. Diese Tumoren treten vornehmlich bei Frauen in perimenopausalem Alter auf [6]. Makroskopisch gibt es ein breites Spektrum von mehr soliden Tumoren bis zu Tumoren, die ausschließlich aus multiplen Zysten bestehen. Die Zysten werden ausgekleidet von Zylinderepithel bzw. kubischem Epithel, die auch kleine Papillen bilden können. In den soliden Arealen finden sich dafür häufig mikrozystische Veränderungen. Das Stroma dieser
Tumoren besteht aus Spindelzellen mit plumpen Nukleolen. Teilweise findet sich ein myxoides Stroma und einzelne Faszikel mit glatten Muskelzellen. Gelegentlich trifft man Fettzellen an $[7,8]$.

In jüngster Zeit wurde die Beziehung zwischen dem zystischen Nephrom und dem gemischten Epithel- und Stromatumor diskutiert. Es wurde vorgeschlagen, den Begriff „renaler Epithel- und Stromatumor" (REST) als vereinheitlichende Bezeichnung für das zystische Nephrom und den gemischten Epithel- und Stromatumor (MEST) zu benutzen [9].

\section{Tubulozystisches Nierenzellkarzinom}

In den letzten Jahren wurden verschiedene Fallberichte über das so genannte tubulozystische Nierenzellkarzinom veröffentlicht [10, 11]. Dieser Tumortyp ist noch nicht in der WHO-Klassifikation 2004 enthalten. Einige dieser Tumoren wurden ursprünglich als niedrig maligne Sammelrohrkarzinome publiziert. Auch diese Tumoren haben eine charakteristische Makroskopie. Die Tumoren sind scharf begrenzt. Die Schnittfläche zeigt ein „Schwamm-artiges“ Aussehen, bestehend aus zahlreichen kleinen Zysten, die mit einer klaren Flüssigkeit angefüllt sind. Mitunter weisen die Zysten eine unterschiedliche Größe auf, jedoch zeigt sich im Wesentlichen ein homogenes Bild. Die Zysten sind mit einer einzelnen Zellschicht ausgekleidet. Die Zellen zeigen ein eosinophiles Zytoplasma. Die Kerne weisen prominente Nukleolen auf. Nekrosen und Mitosefiguren sind selten. 

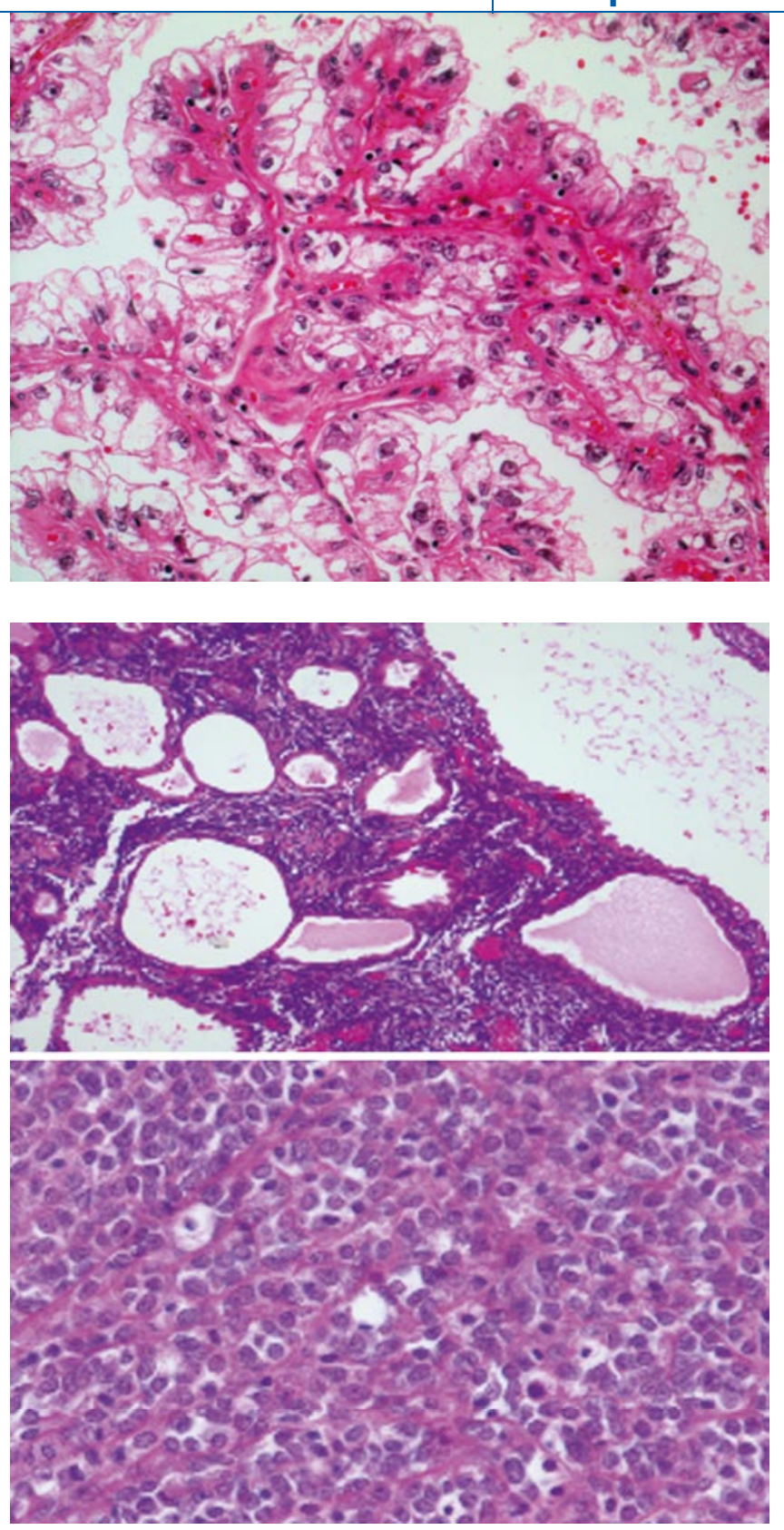

Abb. $1<$ Translokationskarzinom der Niere. Dieser Tumor tritt vorwiegend im Kindesalter auf (HE-Färbung)

Abb. $2 \varangle$ Synoviales Sarkom der Niere. Das zystische Wachstum (oben) ist typisch für diese Tumoren (HE-Färbung)

\section{Nierenkarzinome in erworbenen Zystennieren}

In Endstadium-Schrumpfnieren von Patienten mit chronischer Hämodialysebehandlung oder nach erfolgreicher Nierentransplantation entwickeln sich häufig sekundär entstandene Zysten sowie gelegentlich tubulopapilläre Nierenadenome. In Endstadium-Nieren können prinzipiell alle bekannten Nierentumortypen auftreten, z. B. klarzellige, chromophobe und papilläre Nierenzellkarzinome. Es ist bekannt, dass in Endstadium-Nieren papilläre Karzinome häufiger sind als in NeNierenzellkarzinomen [12].

Eine detaillierte Analyse der Tumortypen in diesen Endstadium-Schrumpfnieren mit erworbenen Nierenzysten hat unlängst gezeigt, dass es sich bei diesen papillären Tumoren teilweise nicht um die typischen papillären Nierenzellkarzinome vom Typ 1 oder 2 handelt, sondern um morphologisch und molekulargenetisch unterschiedliche Karzinomtypen [13]. Einige dieser speziellen Tumortypen scheinen ausschließlich in EndstadiumSchrumpfnieren vorzukommen, andere sind möglicherweise auch im spora- phrektomiepräparaten mit sporadischen dischen Kontext anzutreffen. Insgesamt kann man heute davon ausgehen, dass in Endstadium-Schrumpfnieren 2 besondere Nierentumortypen auftreten:

- das so genannte klarzellige und papilläre Nierenzellkarzinom der Schrumpfniere und

- das Nierenzellkarzinom in erworbenen Zystennieren (,acquired cystic disease-associated renal cell carcinoma").

Der letztgenannte Nierentumortyp tritt nur in erworbenen Zystennieren auf und zeigt eine solide homogene, manchmal mikrozystische Schnittfläche. Das Nierenzellkarzinom in erworbenen Zystennieren hat großvolumige, zytoplasmareiche $\mathrm{Tu}$ morzellen mit eosinophilem Zytoplasma und Fuhrman-Grad-3-Kernen. Fokal kann eine papilläre Architektur bestehen. Oxalatkristalle sind häufig [14].

Der zweite Tumortyp, das klarzellige und papilläre Nierenzellkarzinom der Schrumpfniere kann sowohl in erworbenen Zystennieren, aber auch in "nichtzystischen" Endstadium-Schrumpfnieren angetroffen werden. Die mikroskopische Untersuchung zeigt manchmal ein Wachstum „wie in einer Zyste“. Daher wurde unlängst auch der Begriff des „klarzelligen und papillären und zystischen Nierenzellkarzinoms" vorgeschlagen. Mikroskopisch besteht eine charakteristische papilläre Struktur, die Nierenkarzinomzellen zeigen jedoch das Zytoplasma von klarzelligen Nierenkarzinomen. Diagnostisch wegweisend ist in diesen Fällen die klare papilläre Morphologie mit einem ungewöhnlich hellen Zytoplasma und einem niedrigen Differenzierungsgrad (Fuhrman-Grad 1 bis 2). Solche Tumoren werden mittlerweile auch im sporadischen Kontext beschrieben.

\section{Klarzelliges Nierenzellkarzinom mit prominenten Zysten/ multilokuläres zystisches Nierenzellkarzinom}

Multiple Zysten finden sich in weniger als $5 \%$ der sporadischen klarzelligen Nierenzellkarzinome [15]. Solche Tumoren sind meist scharf begrenzt und haben Zysten mit irregulären, dicken, fibrösen Septen. Diese Zystenbildung in sporadischen 
klarzelligen Nierenkarzinomen muss abgegrenzt werden vom so genannten multilokulären zystischen Nierenzellkarzinom. Jüngere Untersuchungen haben darauf hingewiesen, dass es sich bei diesem Tumortyp um einen Subtyp des klarzelligen Nierenzellkarzinoms handelt [16]. Bei diesen Tumoren finden sich schon makroskopisch zahlreiche multilokuläre Zysten. Die Septen sind sehr dünn. Sobald solide Areale mit hellen Tumorzellen vorkommen, sollten diese Tumoren als klarzellige Karzinome diagnostiziert werden.

Multilokuläre zystische Nierenzellkarzinome haben im Allgemeinen einen niedrigen Differenzierungsgrad (Fuhrman-Grad 1 bis 2). Bei konventionellen Diagnosekriterien wurden bislang keine Metastasen bei diesen Tumoren beschrieben. Teilweise sind diese Tumoren schwierig von multilokulären Zysten abzugrenzen. Diagnostisch wichtiges Kriterium ist hier der Nachweis von epithelialen Tumorzellen innerhalb der fibrösen Septen.

\section{TFE3-Translokationskarzinome}

Dieser Tumortyp wurde erst vor Kurzem beschrieben und tritt im Wesentlichen im Kindes- bzw. jungen Erwachsenenalter auf [17]. Die meisten Patienten haben ein Alter zwischen 4 und 19 Jahren. Eine von uns durchgeführte Analyse von Nierentumoren im Kindes- und jungen Erwachsenenalter unter Einbezug von Fällen aus dem Kindertumorreferenzzentrum in Kiel hat gezeigt, dass dieser Tumortyp in diesem Alter vergleichsweise häufig ist [18].

Ungewöhnlich für diesen Tumortyp ist ein papilläres Wachstum, wobei die Zellen ein klarzelliges Zytoplasma aufweisen (• Abb. 1). Insofern müssen diese Tumoren von den oben erwähnten klarzelligen und papillären Nierenzellkarzinomen in der Endstadium-Niere abgegrenzt werden.

In einer Untersuchung dieses Tumortyps im Erwachsenenalter hat sich gezeigt, dass es sich in diesem Alter um sehr aggressive Tumoren handelt [19]. In einem Fall konnten wir nachweisen, dass über mehrere Jahre eine Zyste vorgelegen hatte, was darauf hinweist, dass auch diese Tumoren durch eine charakteristische Zystenbildung gekennzeichnet sind. Die Tu-

Pathologe 2010 · [Suppl 2] 31:239-243 DOI 10.1007/s00292-010-1317-y

(c) Springer-Verlag 2010

H. Moch

Zystische Nierentumoren. Neue Tumortypen und aktuelle molekulare Erkenntnisse

\section{Zusammenfassung}

Nierentumoren mit makroskopisch prominenter Zystenbildung umfassen ein breites Spektrum bekannter, aber auch weniger bekannter und neuer Nierentumorentitäten. Bekannte Nierentumoren mit dominanter Zystenbildung schließen das zystische Nephrom, den gemischten Epithel- und Stromatumor, das synoviale Sarkom und das multilokuläre zystische Nierenzellkarzinom ein (WHO-Klassifikation 2004). In jüngster Zeit wurden neue Tumortypen beschrieben, die ebenfalls durch eine markante Zystenbildung charakterisiert sind. Beispiele sind das tubulozystische Karzinom und Nierenkarzinome in EndstadiumNieren. Durch ihren charakteristischen Phänotyp und ihr biologisches Verhalten werden diese Nierentumortypen sehr wahrscheinlich in eine zukünftige WHO-Klassifikation als eigene Subtypen aufgenommen werden. Bei Patienten mit von-Hippel-Lindau-Syndrom

\section{Cystic renal neoplasms. New entities and molecular findings}

\section{Abstract}

Renal neoplasms with dominant cysts represent a broad spectrum of known as well as novel renal tumor entities. Established renal tumors with dominant cysts include cystic nephroma, mixed epithelial and stromal tumor, synovial sarcoma and multilocular cystic renal cancer (WHO classification 2004). Novel tumor types have recently been reported, which are also characterized by marked cyst formation. Examples are tubulocystic renal cancer and renal cancer in end-stage renal disease. These tumors are very likely to be included in a future WHO classification due to their characteristic phenotype and molecular features. Cysts and clear cell renal cell carcinoma frequently coexist in the kidneys of pa- treten neben Zysten multiple klarzellige Nierenzellkarzinome auf. Auch sporadische klarzellige Nierenzellkarzinome enthalten oftmals Zysten. Das multilokuläre zystische Nierenzellkarzinom besteht nahezu ausschließlich aus Zysten und wird mittlerweile als besonderer Subtyp des klarzelligen Nierenzellkarzinoms angesehen. Jüngere molekulare Untersuchungen postulieren beim von-Hippel-Lindau-Syndrom einen zystenabhängigen molekularen Tumorentstehungsmechanismus, während bei sporadischen klarzelligen Nierenzellkarzinomen ein so genannter zystenunabhängiger Entstehungsmechanismus anzunehmen ist.

Schlüsselwörter

Multilokuläres zystisches Nierenkarzinom . Tubulozystisches Nierenzellkarzinom . Nierenzysten · Zilien $\cdot$ VHL-Gen tients with von Hippel-Lindau disease. Cysts are also a component of many sporadic clear cell renal cell carcinomas. Multilocular cystic renal cell carcinoma is composed almost exclusively of cysts and is regarded as a specific subtype of clear cell renal cancer. Recent molecular findings suggest that clear cell renal cancer may develop via a cyst-dependent mechanism in von Hippel-Lindau syndrome as well as via cyst-independent molecular pathways in sporadic clear cell renal cancer.

\section{Keywords}

Multilocular renal cancer - Tubulocystic renal cancer · Renal cysts · Cilia · VHL gene 


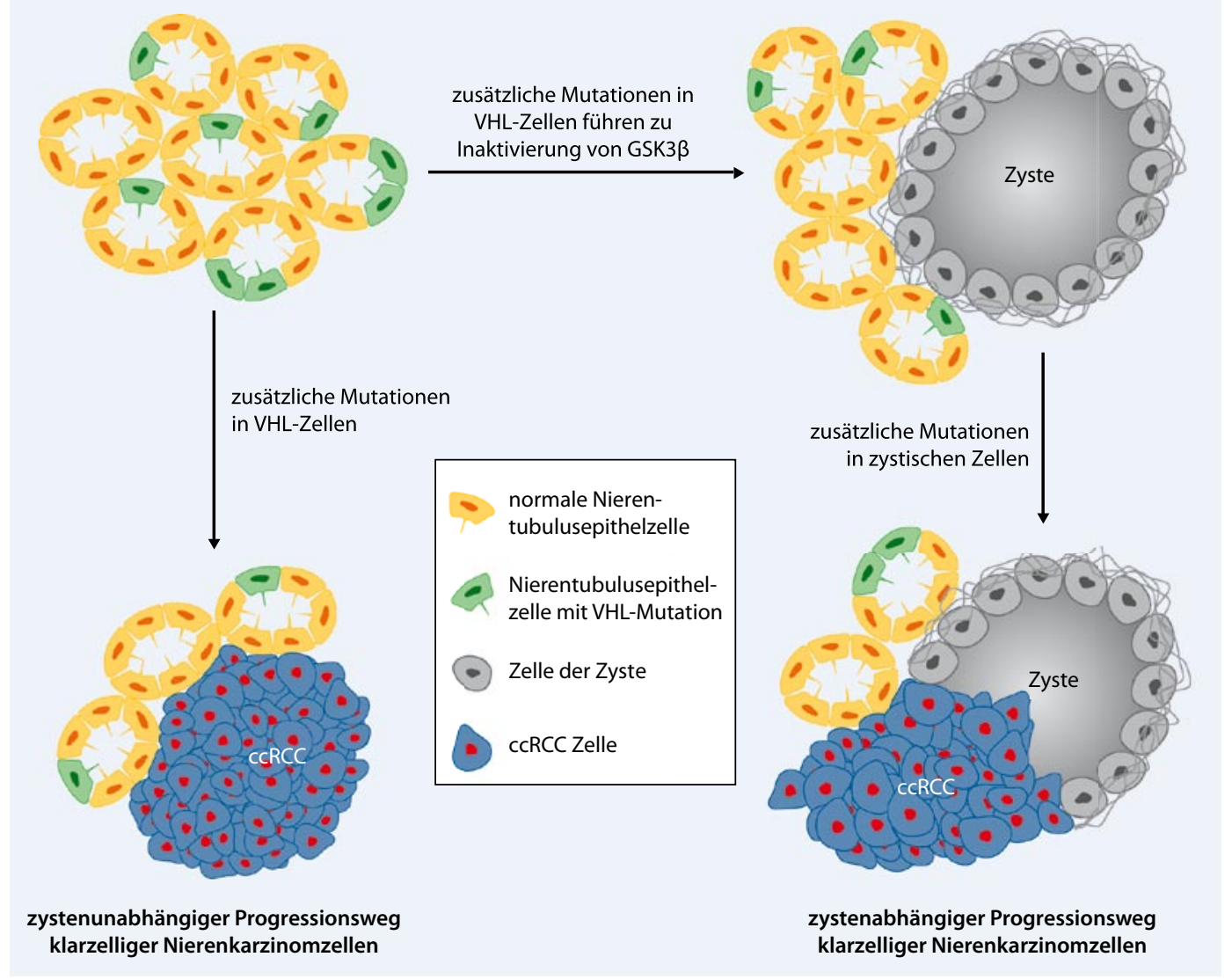

Abb. $3 \triangleleft$ Konzept der Tumorentstehung des klarzelligen Nierenzellkarzinoms über zystische oder nichtzsystische Vorläuferläsionen $\left(c c R C C_{\text {"I }}\right.$ clear cell renal cell carcinoma"). (Adaptiert nach [3])

moren haben unterschiedliche Translokationen, die jedoch alle in eine Genfusion des Gens TFE 3 münden. Häufige Translokationen sind auch $\mathrm{t}(\mathrm{X} ; 1)(\mathrm{p} 11.2 ; \mathrm{q} 21)$ sowie die $\mathrm{t}(\mathrm{X} ; 17)(\mathrm{p} 11.2 ; \mathrm{q} 25)$. Diese resultieren in einer Fusion des $A S P L$ - und $T F E_{3}-G e n s$.

\section{Primäres synoviales Sarkom}

Auch das primäre synoviale Sarkom der Niere kann durch große Zysten imponieren. Dieses Bild führte zur primären Beschreibung als „zystisches embryonales Sarkom der Niere“. Mikroskopisch ist das synoviale Sarkom durch plumpe Spindelzellen charakterisiert. Die Zysten sind mit mitotisch inaktiven Epithelzellen ohne wesentliche zelluläre Atypien ausgeklei$\operatorname{det}$ (• Abb. 2). Die Spindelzellen sind immunreaktiv für EMA, CD56 und teilweise CD99. Das Zystenepithel ist Zytokeratin-positiv.

Bekanntermaßen ist das synoviale Sarkom zytogenetisch charakterisiert durch die Translokation $\mathrm{t}(\mathrm{X} ; 18)(\mathrm{p} 11.27 ; \mathrm{q11.2})$. Molekulardiagnostisch kann im primären synovialen Sarkom der Niere die charakteristische SYT-SSX-Genfusion nachgewiesen werden $[20,21]$. Im Gegensatz zu den synovialen Sarkomen im Weichgewebe ist das primäre synoviale Sarkom der Niere überwiegend durch die SYT-SSX2Genfusion charakterisiert. Möglicherweise resultiert daraus die Tendenz, dass in der Niere nahzu ausschließlich monophasische synoviale Sarkome auftreten.

\section{Zysten als Vorläuferläsion des Nierenzellkarzinoms bei von-Hippel-Lindau-Syndrom}

Bei den verschiedenen histologischen $\mathrm{Tu}$ mortypen wird von unterschiedlichen molekularen Veränderungen ausgegangen, die für die Tumorentstehung verantwortlich sind. So unterscheiden sich papilläre und chromophobe Karzinome sowie Onkozytome [22, 23] von den klarzelligen Karzinomen im Wesentlichen durch das Fehlen der VHL-Gen-Alteration. Die Tatsache, dass in Nieren von Patienten mit von-Hippel-Lindau- (VHL-) Syndrom klarzellige Nierenzellkarzinome und Zysten nebeneinander vorkommen, und das unterschiedliche Ausmaß von Zysten innerhalb von Nierenkarzinomen bei sporadischen Karzinomen hat verschiedene Untersuchungen ausgelöst, die die Bedeu- tung des VHL-Proteins als Bestandteil der Primärzilien der Nierentubulusepithelzellen zeigten [24, 25].

Die Inaktivierung des $V H L$-Gens führt zu einem Verlust dieser Zilien, was die Ausbildung von Zysten begünstigt [26]. Die Reduktion der Zilien wiederum aktiviert Signalwege, die die Proliferation der Nierentubulusepithelzellen beschleunigen [3]. Dieser Vorgang ist möglicherweise einer der ersten Schritte in der Tumorentstehung bei Patienten mit VHLSyndrom. Vor Kurzem konnten wir zeigen, dass tatsächlich in den Epithelzellen der Zysten und auch in Einzelzellen mit CAIX-Expression Deletionen des $V H L$ Gens sowie eine deutliche Veränderung der Zilienmorphologie nachweisbar sind [27]. Damit wird ein neues Modell unterstützt, bei dem ein VHL-induzierter zystenabhängiger und ein zystenunabhängiger Entstehungsmechanismus diskutiert werden (• Abb. 3).

Sporadische und familiäre, VHL-assoziierte Nierenzellkarzinome unterscheiden sich in ihrem Metstasierungsverhalten. Neben den charakteristischen Veränderungen bei der Tumorentstehung sind jedoch zahlreiche und komplexe weitere 
Signalwege für die Erklärung des unterschiedlichen Metastasierungsverhalten heranzuziehen $[28,29,30]$.

\section{Fazit für die Praxis}

Makroskopisch und radiologisch können sich Nierentumoren mit einer markanten Zystenbildung präsentieren. Für die Praxis der Radiologie und Pathologie ist allerding bedeutsam, dass zystische Nierentumoren eine sehr gute Prognose (zystisches Nephrom/gemischter Epithel- und Stromatumor, multilokuläres zystisches Nierenzellkarzinom, zystisches Onkozytom), aber auch hochmaligne (z. B. synoviales Sarkom, Translokationskarzinom und andere) sein können. In der nächsten WHO-Klassifikation werden sehr wahrscheinlich weitere zystische Nierentumortypen (tubulozystisches Nierenzellkarzinom, Nierenkarzinome in Endstadium-Nieren) berücksichtigt werden.

\section{Korrespondenzadresse \\ Prof. Dr. H. Moch \\ Institut für Klinische Pathologie, \\ UniversitätsSpital Zürich \\ Schmelzbergstr. 12, 8091 Zürich \\ Schweiz \\ holger.moch@usz.ch}

Interessenkonflikt. Der korrespondierende Autor gibt an, dass kein Interessenkonflikt besteht.

\section{Literatur}

1. Fine SW, Reuter VE, Epstein Jl, Argani P (2006) Angiomyolipoma with epithelial cysts (AMLEC): a distinct cystic variant of angiomyolipoma. Am J Surg Pathol 30(5):593-599

2. Amin MB, Crotty TB, Tickoo SK, Farrow GM (1997) Renal oncocytoma: a reappraisal of morphologic features with clinicopathologic findings in 80 cases. Am J Surg Pathol 21(1):1-12

3. Thoma CR, Frew IJ, KrekW (2007) The VHL tumor suppressor: riding tandem with GSK3beta in primary cilium maintenance. Cell Cycle 6(15):18091813

4. Moch $\mathrm{H}$ (2010) Cystic renal tumors: new entities and novel concepts. Adv Anat Pathol 17(3):209214

5. Pawade J, Soosay GN, Delprado W et al (1993) Cystic hamartoma of the renal pelvis. Am J Surg Pathol 17(11):1169-1175

6. Adsay NV, Eble JN, Srigley JR et al (2000) Mixed epithelial and stromal tumor of the kidney. Am J Surg Pathol 24(7):958-970

7. Michal M, Syrucek M (1998) Benign mixed epithelial and stromal tumor of the kidney. Path Res Pract 194:445-448
8. Moch H, Schurch LV, Sulser T, Terracciano L (2004) Mixed epithelial and stromal tumor of the kidney. Pathologe 25(5):356-361

9. Antic T, Perry KT, Harrison K et al (2006) Mixed epithelial and stromal tumor of the kidney and cystic nephroma share overlapping features: reappraisal of 15 lesions. Arch Pathol Lab Med 130(1):80-85

10. Amin MB, MacLennan GT, Gupta R et al (2009) Tubulocystic carcinoma of the kidney: clinicopathologic analysis of 31 cases of a distinctive rare subtype of renal cell carcinoma. Am J Surg Pathol 33(3):384-392

11. Zhou M, Yang XJ, Lopez Jl et al (2009) Renal tubulocystic carcinoma is closely related to papillary renal cell carcinoma: implications for pathologic classification. Am J Surg Pathol 33(12):1840-1849

12. Moch H, Urrejola C, Herrmann P et al (1997) Nierentumoren bei Langzeit-Hämodialyse und in Schrumpfnieren. In: Lison A (Hrsg) Werkstattgespräch Nephrologie 1997. Pabst Science Publishers, Lengerich Berlin Düsseldorf Leipzig Riga Scottsdale/AZ (USA) Wien Zagreb, S 121-132

13. Tickoo SK, Deperalta-Venturina MN, Harik LR et al (2006) Spectrum of epithelial neoplasms in endstage renal disease: an experience from 66 tumorbearing kidneys with emphasis on histologic patterns distinct from those in sporadic adult renal neoplasia. Am J Surg Pathol 30(2):141-153

14. Sule N, Yakupoglu U, Shen SS et al (2005) Calcium oxalate deposition in renal cell carcinoma associated with acquired cystic kidney disease: a comprehensive study. Am J Surg Pathol 29(4):443-451

15. Eble JN, Bonsib SM (1998) Extensively cystic renal neoplasms: cystic nephroma, cystic partially differentiated nephroblastoma, multilocular cystic renal cell carcinoma, and cystic hamartoma of renal pelvis. Semin Diagn Pathol 15(1):2-20

16. Halat S, Eble JN, Grignon DJ et al (2010) Multilocular cystic renal cell carcinoma is a subtype of clear cell renal cell carcinoma. Mod Pathol 23(7):931936

17. Argani $P$, Antonescu CR, Illei PB et al (2001) Primary renal neoplasms with the ASPL-TFE3 gene fusion of alveolar soft part sarcoma: a distinctive tumor entity previously included among renal cell carcinomas of children and adolescents. Am J Pathol 159(1):179-192

18. Bruder E, Passera O, Harms D et al (2004) Morphologic and molecular characterization of renal cell carcinoma in children and young adults. Am J Surg Pathol 28(9):1117-1132

19. Argani P, Olgac S, Tickoo SK et al (2007) Xp11 translocation renal cell carcinoma in adults: expanded clinical, pathologic, and genetic spectrum. Am J Surg Pathol 31(8):1149-1160

20. Argani P, Faria PA, Epstein Jl et al (2000) Primary renal synovial sarcoma: molecular and morphologic delineation of an entity previously included among embryonal sarcomas of the kidney. Am J Surg Pathol 24(8):1087-1096

21. Moch H, Wodzynski A, Guillou L, Nickeleit V (2003) Primary renal synovial sarcoma. A new entity in the morphological spectrum of spindle cell renal tumors. Pathologe 24(6):466-472

22. Bugert P, Kovacs G (1996) Molecular differential diagnosis of renal cell carcinomas by microsatellite analysis. Am J Pathol 149(6):2081-2088

23. Presti J, Moch H, Reuter V et al (1996) Chromosome 1 and 14 loss in renal oncocytomas. Genes Chromos Cancer 17:199-204

24. Thoma CR, Frew IJ, Hoerner CR et al (2007) pVHL and GSK3beta are components of a primary cilium-maintenance signalling network. Nat Cell Biol 9(5):588-595
25. SchramI P, Frew IJ, Thoma CR et al (2009) Sporadic clear cell renal cell carcinoma but not the papillary type is characterized by severely reduced frequency of primary cilia. Mod Pathol 22(1):31-36

26. Frew IJ, Thoma CR, Georgiev S et al (2008) pV$\mathrm{HL}$ and PTEN tumour suppressor proteins cooperatively suppress kidney cyst formation. Embo J 27(12):1747-1757

27. Montani M, Heinimann K, Teichman A von et al (o J) VHL-gene deletion in single renal tubular epithelial cells and renal tubular cysts: further evidence for a cyst-dependent progression pathway of clear cell renal carcinoma in von Hippel-Lindau disease. Am J Surg Pathol 34(6):806-815

28. Dahinden C, Ingold B, Wild P et al ( $n$ d) Mining tissue microarray data to uncover combinations of biomarker expression patterns that improve intermediate staging and grading of clear cell renal cell cancer. Clin Cancer Res 16(1):88-98

29. Moch H, Sauter G, Buchholz N et al (1997) Epidermal growth factor receptor expression is associated with rapid tumor cell proliferation in renal cell carcinoma. Hum Pathol 28(11):1255-1259

30. Moch H, Sauter G, Gasser T et al (1998) EGF-r gene copy number gains detected by fluorescence in situ hybridization in renal cell carcinoma. J Pathol 184:424-429 